\title{
Effectiveness of a gamification strategy to prevent childhood obesity in schools: a cluster-randomized controlled trial
}

\author{
Sebastián Peña ${ }^{1}$, Macarena Carranza ${ }^{2}$, Cristóbal Cuadrado ${ }^{3}$, Paula Espinoza $^{2}$, Valeska Müller $^{2}$ \\ and Ricardo Cerda ${ }^{4}$ \\ ${ }^{1}$ Department of Public Health Solutions, National Institute for Health and Welfare, Helsinki, Finland, Helsinki, \\ Finland, \\ ${ }^{2}$ Santiago Sano Program, Municipality of Santiago, Santiago, Chile, \\ ${ }^{3}$ Programa de Politicas, Sistemas y Gestión en Salud. Escuela de Salud Pública, Universidad de Chile, Santiago, Chile, \\ Santiago, Chile and \\ ${ }^{4}$ Departamento de Nutrición, Facultad de Medicina, Universidad de Chile, Santiago, Chile, Santiago, Chile
}

\begin{abstract}
Introduction: School-based interventions to prevent obesity have shown heterogeneous results. Recent school-based trials with "negative" have cast doubt on their effectiveness. In the Juntos Santiago trial, we used an innovative, theory-based gamification strategy to increase motivation and participation in healthy behavioural changes. This is the first trial, to our knowledge, to use gamification to prevent childhood obesity.
\end{abstract}

Objective: To examine the effectiveness of a multicomponent gamification strategy to prevent obesity in 5th and 6th-grade schoolchildren in Santiago de Chile.

Materials and methods: School-based, parallel cluster-randomized controlled trial. 81 schools in two municipalities in Santiago, Chile, with more than 40 students in 5th and 6th grade altogether were eligible. Schools were randomized and sequentially invited to participate. The multicomponent intervention was a gamification strategy with four components: (i) healthy challenges (snacks, steps and healthy activities); (ii) gamification incentives, such as points, levels, leaderboards and badges; (iii) rewards (infrastructure and fun activity) and (iv) an online platform for parents and students to monitor the progress. Participants were followed up at 5 and 8 months. Pre-registered primary outcomes were body mass index (BMI) z-score and waist circumference (WC) at 8 months. Secondary outcomes were BMI and systolic (SBP) and diastolic blood pressure (DBP) at 8 months. Multilevel analysis adjusted for individual and school-level covariates were used.

Results: 24 schools ( 9 controls) and 2333 students (709 controls) were assessed at baseline and 2264 students (689 controls) were assessed at 8 months. The mean BMI z-score was lower in the intervention group compared to the control group at 8 months (mean difference $-0.132,95 \%$ CI $-0.249 ;-0.014$ ), while no difference was observed for WC. Mean BMI and SBP were lower in the intervention arm compared to the control arm (mean difference for SBP $-0.139 ; 95 \%$ CI $-2.39 ;-0.40$ ). No difference was observed for DBP.

Discussion: The gamification strategy appears to prevent childhood obesity and reduce systolic blood pressure in school children in Santiago. These effects are larger than the pooled effects from existing meta-analyses. Further research should identify which components of the gamification strategy were more effective.

Trial registration: ClinicalTrials.gov NCT03459742

Funded by: Mayors Challenge 2016, Bloomberg Philanthropies

\section{Conflict of Interest}

There is no conflict of interest 